\title{
Effect of saponified high fat sunflower oilcake and lipoic acid on fat quality of lambs
}

\author{
F.K. Siebrits ${ }^{1 \#}$, A. Makgekgenene ${ }^{1}$ and A. Hugo ${ }^{2}$ \\ ${ }^{1}$ Tshwane University of Technology, Animal Sciences, Private Bag X680, Pretoria 0001, South Africa \\ ${ }^{2}$ University of the Free State, Microbial Biochemical and Food Biotechnology, P.O. Box 339, \\ Bloemfontein 9300, South Africa
}

\begin{abstract}
Sheep fat contains relatively high levels of saturated fatty acids while poly-unsaturated fatty acids (PUFA) are toxic to cellulolytic bacteria and are also saturated in the rumen. Stabilization of residual oil in sunflower oilcake by conversion into calcium salts would be advantageous. Alpha lipoic acid acts as an antioxidant to ameliorate the effects of oxidative stress caused by high dietary levels of PUFA. Residual oil $(14 \%)$ in mechanically extracted (expeller) sunflower oilcake (SFOC) was saponified in situ and compared in a complete feedlot diet ( $100 \mathrm{~g}$ crude protein and $31 \mathrm{~g}$ extractable fat $/ \mathrm{kg}$ feed) with commercially extracted oilcake containing 2.4\% residual oil (control diet with $29 \mathrm{~g}$ fat and $123 \mathrm{~g}$ crude protein $\mathrm{kg}$ feed) fed to four groups of $10 \mathrm{SA}$ Mutton Merino weaner lambs (ca. $23 \mathrm{~kg}$ ) for nine weeks. Both diets were fed either with, or without a weekly oral dosing of $500 \mathrm{mg} \alpha$-lipoic acid. Fatty acid composition was determined on back fat samples while thiobarbituric acid-reactive substances (TBARS) were determined on samples of $m$. longissimus dorsi stored for zero and six months and displayed for six days. The back fat of the lambs on the saponified expeller SFOC diets contained significantly higher levels of saturated fatty acids and lower levels of mono-unsaturated fatty acids. PUFA were unaffected. High TBARS levels $(>1.0)$ were found after six months storage. Non significant increases in TBARS were observed in the groups that received lipoic acid.
\end{abstract}

Keywords: Calcium soap, TBARS, fatty acid composition, shelf life

${ }^{\#}$ Corresponding author. E-mail: siebritsfk@tut.ac.za

\section{Introduction}

Sheep fat generally contains relatively high levels of saturated fatty acids (Okeudo \& Moss, 2007) while polyunsaturated fatty acids (PUFA) are toxic to cellulolytic bacteria and are saturated by hydrogenation in the rumen (Maia et al., 2007). Expeller sunflower oilcake with a relatively high (15\%) residual oil content may become available from envisaged rural small-scale oil presses. Since it is recommended that human diets should contain more mono unsaturated fatty acids (MUFA) to replace saturated fatty acids (Krauss et al., 1998) and the health conscious market requires products with higher PUFA content it could be advantageous to stabilize the residual oil in sunflower oilcake by converting it into calcium salts. High levels of PUFA may cause oxidative stress in sheep. Alpha lipoic acid acts as an antioxidant (Rui-li Yang et al., 2008) to ameliorate the effects of oxidative stress caused by high levels of PUFA. The present study was therefore conducted to determine to what extent the unsaturated fatty acid content of lamb back fat can be manipulated by the feeding of saponified expeller oilcake, what the effect would be on fat stability and shelf life in terms of thiobarbituric acid-reactive substances (TBARS) in the $m$. longissimus dorsi and whether the weekly feeding of $\alpha$-lipoic acid as a powerful anti oxidant would improve stability.

\section{Materials and Methods}

Two complete feedlot diets were formulated, each containing maize meal, wheaten bran, molasses meal with minerals and vitamins and $15 \%$ sunflower oilcake meal (SFOC). The experimental SFOC was mechanically extracted (expeller) containing 14\% residual oil saponified in situ. The control SFOC consisted of commercially extracted oilcake containing $2.4 \%$ residual oil which was not saponified. These diets were fed to four groups of $10 \mathrm{SA}$ Mutton Merino weaner lambs (ca. $23 \mathrm{~kg})$ for nine weeks. Both diets were fed either with, or without weekly oral dosing with $500 \mathrm{mg} \alpha$-lipoic acid. Fatty acid composition was determined on subcutaneous back fat samples collected between the 10th and 12th rib over the m. longissimus dorsi 
while samples of the latter were taken at the same site after 24 hour chilling, vacuum packed and stored at $-18{ }^{\circ} \mathrm{C}$ for determination of thiobarbituric acid-reactive substances (TBARS) using the aqueous acid extraction method of Raharjo et al. (1992). Meat cores were taken from fresh and frozen $\left(-18{ }^{\circ} \mathrm{C}\right.$ for six months) muscle samples, placed on polystyrene trays and covered with an oxygen permeable PVC wrap. Meat samples were displayed at $4{ }^{\circ} \mathrm{C}$ under fluorescent light for six days before TBARS were determined. The fatty acid data and TBARS values were subjected to analysis of variance while differences between treatments were identified by means of least significant differences using StataCorp ${ }^{\circledR}$ (2007).

\section{Results and Discussion}

The back fat fatty acid compositions of the sheep on the four treatments are depicted in Tables 2 and 3.

Table 1 Dietary fatty acid composition of experimental sunflower oil cake meals (SFOC)

\begin{tabular}{lcc}
\hline & Extracted SFOC & Saponified expeller SFOC \\
\hline Fatty acid composition (\% of total fatty acids) & & 21.96 \\
Saturated fatty acids & 19.50 & 37.45 \\
Monounsaturated fatty acids & 36.40 & 40.59 \\
Polyunsaturated fatty acids & 44.10 & 4.80 \\
Trans fatty acids & 0.00 & 70.63 \\
Cis fatty acids & 77.71 & 2.12 \\
Omega 3 fatty acids & 2.28 & 0.00 \\
Omega 6 fatty acids & 0.00 & 0.18 \\
C14 fatty acids & & 15.17 \\
C16 fatty acids & 0.11 & 80.66 \\
C18 fatty acids & 14.49 & 82.29 \\
\hline
\end{tabular}

Table 2 Summary of fatty acids ( $\%$ of total fatty acids) of sheep back fat (mean \pm s.d.)

\begin{tabular}{|c|c|c|c|c|}
\hline & $\begin{array}{c}\text { Extracted SFOC } \\
\& \text { lipoic acid }\end{array}$ & Extracted SFOC & $\begin{array}{c}\text { Saponified } \\
\text { expeller SFOC }\end{array}$ & $\begin{array}{c}\text { Saponified } \\
\text { expeller SFOC } \\
\text { \& lipoic acid }\end{array}$ \\
\hline Saturated fatty acids & $54.96^{\mathrm{b}} \pm 2.27$ & $54.86^{\mathrm{b}} \pm 2.26$ & $58.89^{\mathrm{a}} \pm 3.65$ & $58.23^{\mathrm{ab}} \pm 3.57$ \\
\hline Monounsaturated fatty acids & $40.95 \pm 2.00$ & $40.62 \pm 1.82$ & $37.75 \pm 3.87$ & $37.44 \pm 3.63$ \\
\hline Polyunsaturated fatty acids & $4.08 \pm 1.02$ & $4.51 \pm 2.06$ & $3.36 \pm 0.79$ & $4.33 \pm 1.27$ \\
\hline Trans fatty acids & $2.61 \pm 0.74$ & $2.64 \pm 0.58$ & $2.64 \pm 1.13$ & $2.83 \pm 0.46$ \\
\hline Cis fatty acids & $36.98 \pm 2.01$ & $37.30 \pm 1.78$ & $35.71 \pm 3.36$ & $34.64 \pm 3.34$ \\
\hline $\mathrm{C} 18: 2$ / C:18:3 & $14.13^{\mathrm{ab}} \pm 5.50$ & $9.94^{b} \pm 3.63$ & $16.86^{\mathrm{a}} \pm 6.16$ & $13.41^{\mathrm{ab}} \pm 3.20$ \\
\hline MUFA / saturated & $0.75 \pm 0.07$ & $0.74 \pm 0.05$ & $0.65 \pm 0.10$ & $0.65 \pm 0.10$ \\
\hline Unsaturated / total & $0.45^{\mathrm{a}} \pm 0.02$ & $0.45^{\mathrm{a}} \pm 0.02$ & $0.41^{\mathrm{b}} \pm 0.04$ & $0.42^{\mathrm{ab}} \pm 0.04$ \\
\hline Unsaturated / Saturated & $0.82^{\mathrm{a}} \pm 0.08$ & $0.83^{\mathrm{a}} \pm 0.08$ & $0.70^{\mathrm{b}} \pm 0.10$ & $0.72^{\mathrm{ab}} \pm 0.10$ \\
\hline $\mathrm{C}: 18$ fatty acids & $26.35^{\mathrm{a}} \pm 1.34$ & $26.28^{\mathrm{a}} \pm 2.10$ & $24.67^{\mathrm{b}} \pm 1.87$ & $25.18^{\mathrm{ab}} \pm 1.34$ \\
\hline$C: 16$ fatty acids & $28.00^{\mathrm{a}} \pm 3.39$ & $28.41^{\mathrm{a}} \pm 2.85$ & $34.75^{\mathrm{b}} \pm 5.63$ & $32.83^{\mathrm{ab}} \pm 4.88$ \\
\hline $\mathrm{C}: 14$ fatty acids & $3.06 \pm 0.45$ & $2.81 \pm 0.31$ & $2.72 \pm 0.40$ & $2.90 \pm 0.21$ \\
\hline$n-6: n-3$ ratio & $2.76^{b} \pm 1.09$ & $3.96^{b} \pm 3.39$ & $19.08^{\mathrm{a}} \pm 10.2$ & $4.30^{\mathrm{b}} \pm 2.33$ \\
\hline
\end{tabular}

$\overline{a, b}$ Values in rows with different superscripts differ significantly $(\mathrm{P} \leq 0.05)$. SFOC - sunflower oil cake.

The diets contained about $80 \%$ unsaturated fatty acids and $20 \%$ saturated fatty acids (Table 1) while sheep fat contained about $55-59 \%$ saturated fatty acids (Table 2) and only $3.4-4.5 \%$ PUFA. Furthermore, the lambs receiving diets containing saponified expeller SFOC had significantly $(\mathrm{P} \leq 0.05)$ higher levels of 
saturated fatty acids than the lambs on the diet containing extracted (unsaponified) SFOC while the MUFA and PUFA levels were not significantly different. This was an unexpected result since the saponified expeller oilcake diet was supposed to contain rumen protected PUFA which could alter the back fat fatty acid composition. Arana et al. (2006) found that saponified olive oil could not alter the PUFA content of sheep fat and in fact decreased it. Kowalski (1997) also found that the calcium soap of rapeseed fatty acids was extensively biohydrogenated. The saponified SFOC caused the lambs to have a significantly $(\mathrm{P} \leq 0.05)$ higher ratio of C18:2/C:18:3 fatty acids also suggesting rumen biohydrogenation. Polyunsaturated fatty acid concentrations were unaffected by treatment. Lipoic acid had no effect on fatty acid composition or saturation but significantly $(\mathrm{P} \leq 0.05)$ decreased the ratio of $n-6: n-3$ fatty acids in the saponified diet. This was not due to a significant change in any of the n-3 or n-6 fatty acids (Table 3) but rather due to small shifts with a cumulative effect on the ratio. The MUFA content of sheep back fat was unaffected by saponification. The dosing with lipoic acid had no significant effect on back fat fatty acid composition.

Table 3 Mean fatty acid composition ( $\%$ of total fatty acids) of sheep back fat ( \pm s.d.)

\begin{tabular}{lcccc}
\hline & $\begin{array}{c}\text { Extracted SFOC \& } \\
\text { lipoic acid }\end{array}$ & Extracted SFOC & $\begin{array}{c}\text { Saponified expeller } \\
\text { SFOC }\end{array}$ & $\begin{array}{c}\text { Saponified } \\
\text { expeller SFOC \& } \\
\text { lipoic acid }\end{array}$ \\
\hline C10:0 & $0.12^{\mathrm{b}} \pm 0.01$ & $0.13^{\mathrm{b}} \pm 0.05$ & $0.13^{\mathrm{b}} \pm 0.02$ & $0.18^{\mathrm{a}} \pm 0.05$ \\
$\mathrm{C} 12: 0$ & $0.10 \pm 0.01$ & $0.10 \pm 0.01$ & $0.10 \pm 0.02$ & $0.11 \pm 0.01$ \\
$\mathrm{C} 14: 0$ & $2.97 \pm 0.43$ & $2.73 \pm 0.29$ & $2.61 \pm 0.4$ & $2.83 \pm 0.23$ \\
$\mathrm{C} 14: 1$ & $0.09 \pm 0.03$ & $0.08 \pm 0.03$ & $0.11 \pm 0.05$ & $0.07 \pm 0.03$ \\
$\mathrm{C} 15: 0$ & $0.55 \pm 0.09$ & $0.52 \pm 0.04$ & $0.51 \pm 0.09$ & $0.55 \pm 0.13$ \\
$\mathrm{C} 16: 0$ & $24.75 \pm 1.33$ & $24.72 \pm 1.94$ & $23.52 \pm 1.75$ & $23.88 \pm 1.19$ \\
$\mathrm{C} 16: 1$ & $1.60^{\mathrm{a}} \pm 0.24$ & $1.56^{\mathrm{a}} \pm 0.24$ & $1.15^{\mathrm{b}} \pm 0.19$ & $1.30^{\mathrm{b}} \pm 0.24$ \\
$\mathrm{C} 18: 0$ & $23.76^{\mathrm{b}} \pm 2.66$ & $23.62^{\mathrm{b}} \pm 2.22$ & $29.60^{\mathrm{a}} \pm 5.02$ & $27.71^{\mathrm{ab}} \pm 4.65$ \\
$\mathrm{C} 18: 1 \mathrm{n} 9 \mathrm{t}$ & $2.37 \pm 0.72$ & $2.45 \pm 0.57$ & $2.23 \pm 1.13$ & $2.37 \pm 0.46$ \\
$\mathrm{C} 18: 1 \mathrm{n} 9 \mathrm{c}$ & $35.48 \pm 1.98$ & $35.40 \pm 1.71$ & $33.37 \pm 3.78$ & $32.55 \pm 3.43$ \\
$\mathrm{C} 18: 2 \mathrm{n} 6 \mathrm{t}$ & $0.24^{\mathrm{b}} \pm 0.07$ & $0.19^{\mathrm{b}} \pm 0.10$ & $0.41^{\mathrm{a}} \pm 0.11$ & $0.46^{\mathrm{a}} \pm 0.07$ \\
$\mathrm{C} 18: 2 \mathrm{n} 6 \mathrm{c}$ & $1.50^{\mathrm{b}} \pm 0.33$ & $1.90^{\mathrm{ab}} \pm 1.03$ & $2.34^{\mathrm{a}} \pm 0.54$ & $2.09^{\mathrm{ab}} \pm 0.20$ \\
$\mathrm{C} 18: 3 \mathrm{n} 6$ & $0.03 \pm 0.01$ & $0.07 \pm 0.04$ & $0.07 \pm 0.04$ & $0.08 \pm 0.05$ \\
$\mathrm{C} 18: 3 \mathrm{n} 3$ & $0.13 \pm 0.05$ & $0.19 \pm 0.20$ & $0.11 \pm 0.02$ & $0.12 \pm 0.02$ \\
$\mathrm{C} 20: 0$ & $0.19^{\mathrm{b}} \pm 0.04$ & $0.21^{\mathrm{b}} \pm 0.06$ & $0.24^{\mathrm{b}} \pm 0.07$ & $0.32^{\mathrm{a}} \pm 0.08$ \\
$\mathrm{C} 20: 1$ & $0.13 \pm 0.09$ & $0.08 \pm 0.02$ & $0.07 \pm 0.01$ & $0.11 \pm 0.04$ \\
$\mathrm{C} 20: 2$ & $0.25^{\mathrm{a}} \pm 0.14$ & $0.26^{\mathrm{a}} \pm 0.14$ & $0.06^{\mathrm{b}} \pm 0.04$ & $0.23^{\mathrm{ab}} \pm 0.23$ \\
$\mathrm{C} 22: 0$ & $0.76 \pm 0.21$ & $1.20 \pm 0.61$ & $0.54 \pm 0.46$ & $0.94 \pm 0.94$ \\
$\mathrm{C} 15: 1$ & $0.27 \pm 0.08$ & $0.25 \pm 0.09$ & $0.23 \pm 0.06$ & $0.28 \pm 0.08$ \\
$\mathrm{C} 17: 0$ & $1.95 \pm 0.44$ & $1.95 \pm 0.32$ & $2.00 \pm 0.35$ & $1.98 \pm 0.42$ \\
$\mathrm{C} 17: 1$ & $0.73 \pm 0.17$ & $0.69 \pm 0.11$ & $0.54 \pm 0.14$ & $0.55 \pm 0.21$ \\
$\mathrm{C} 20: 3 \mathrm{n} 6$ & $0.42^{\mathrm{a}} \pm 0.23$ & $0.37^{\mathrm{ab}} \pm 0.21$ & $0.09^{\mathrm{b}} \pm 0.05$ & $0.21^{\mathrm{ab}} \pm 0.24$ \\
$\mathrm{C} 20: 4 \mathrm{n} 6$ & $0.20 \pm 0.09$ & $0.29 \pm 0.26$ & $0.08 \pm 0.04$ & $0.19 \pm 0.18$ \\
$\mathrm{C} 20: 5 \mathrm{n} 3$ & $0.76 \pm 0.57$ & $0.88 \pm 0.90$ & $0.26 \pm 0.24$ & $0.64 \pm 0.41$ \\
$\mathrm{C} 24: 1$ & $0.11 \pm 0.04$ & $0.11 \pm 0.05$ & $0.09 \pm 0.04$ & $0.20 \pm 0.21$ \\
\hline
\end{tabular}

${ }^{\mathrm{a}, \mathrm{b}}$ Values in rows with different superscripts differ significantly $(\mathrm{P} \leq 0.05)$. SFOC - sunflower oil cake.

The TBARS values as an indication of lipid oxidation are presented in Table 4. High TBARS levels $(>1.0)$ were found after six months storage (Table 4). Neither diet nor Lipoic acid had a significant effect on obtained TBARS values, which corresponds with the results of Schmidt et al. (2005). The tendency was actually for the TBARS levels to be higher in the groups dosed with lipoic acid (500 mg/sheep per week). Likewise Vignola et al. (2009) could not find a change in TBARS by feeding different selenium sources at different levels. Wood et al. (2008) reported that ruminants often have higher values $(2.0-6.2 \mathrm{mg} / \mathrm{kg})$ than pork $(0.6 \mathrm{mg} / \mathrm{kg})$ despite their higher contents of saturated fatty acids. 
Table 4 Mean total thiobarbituric acid-reactive substances (TBARS) (mg/kg muscle) in sheep longissimus dorsi muscles determined on fresh samples, samples stored for six days or for six months ( \pm s.d.)

\begin{tabular}{lcccc}
\hline \multicolumn{1}{c}{ Time } & $\begin{array}{c}\text { Extracted SFOC \& } \\
\text { lipoic acid }\end{array}$ & Extracted SFOC & $\begin{array}{c}\text { Saponified expeller } \\
\text { SFOC }\end{array}$ & $\begin{array}{c}\text { Saponified expeller } \\
\text { SFOC \& lipoic acid }\end{array}$ \\
\hline Fresh & $0.105^{\mathrm{b}} \pm 0.03$ & $0.091^{\mathrm{b}} \pm 0.01$ & $0.090^{\mathrm{c}} \pm 0.05$ & $0.102^{\mathrm{c}} \pm 0.01$ \\
6 Days & $0.611^{\mathrm{a}} \pm 1.09$ & $0.383^{\mathrm{a}} \pm 0.47$ & $0.327^{\mathrm{b}} \pm 0.15$ & $0.574^{\mathrm{b}} \pm 0.42$ \\
6 Months & $1.903^{\mathrm{a}} \pm 1.68$ & $1.396^{\mathrm{a}} \pm 0.97$ & $1.953^{\mathrm{a}} \pm 1.11$ & $2.569^{\mathrm{a}} \pm 1.30$
\end{tabular}

$\overline{\mathrm{a}, \mathrm{b}}$ Values in columns with different superscripts differ significantly $(\mathrm{P} \leq 0.05)$. Treatment effects were not significant. SFOC - sunflower oil cake.

It can be concluded that saponification of residual oil in sunflower oilcake did not achieve its protection against rumen biohydrogenation and that it actually enhanced the process, possibly because the fatty acid salts were in the free form and not bound in triacyl glycerol molecules. Saponification to modify fat composition in sheep is therefore ineffective. Even though there wasn't increased PUFA in the back fat of any of the treatments, no explanation for the absence of an antioxidant effect on the TBARS results can be offered, except perhaps that the dosage or the frequency of dosage was inadequate. However, the tendency was in the wrong direction.

\section{Acknowledgements}

Thanks to K-J. Leeuw and L.S. Maesela of the ARC Irene for the technical support. Thanks are due to Mr Maupi Letsoalo for statistical support. Funding (GUN2069289) of the National Research Foundation is gratefully acknowledged.

\section{References}

Arana, A., Mendizabal, J.A., Alzón, M., Eguinoa, P., Beriain, M.J. \& Purroy, A., 2006. Effect of feeding lambs oleic acid calcium soaps on growth, adipose tissue development and composition. Small Rumin. Res. 63, 75-83.

Kowalski, Z.M., 1997. Rumen fermentation, nutrient flow to the duodenum and digestibility in bulls fed calcium soaps of rapeseed fatty acids and soya bean meal coated with calcium soaps. Anim. Feed Sci. Technol. 69, 289-303.

Krauss, R.M., Winston, M., Fletcher, B.J. \& Grundy, S.M., 1998. Obesity: Impact on Cardiovascular Disease. Circulation 98, 1472-1476.

Maia, M.R.G., Chaudhary, L.C., Figueres, L. \& Wallace, R.J., 2007. Metabolism of polyunsaturated fatty acids and their toxicity to the microflora of the rumen. Antonie van Leeuwenhoek 91, 303-314.

Okeudo, N.J. \& Moss, B.W., 2007. Intramuscular lipid FA profile of sheep comprising four sex-types and seven slaughter weights produced following commercial procedure. Meat Sci. 76, 195-200.

Raharjo, S., Sofos, J.N.S. \& Schmidt, G.R., 1992. Improved speed specificity, and limit of determination of an aqueous acid extraction thiobarbituric acid-C18 method for measuring lipid peroxidation in beef. J. Agric. Food Chem. 40, 2182-2185.

Rui-li Yang, M.S., Wu Li, M.S., Yong-Hui Shi, M.S. \& Guo-Wei Le, Ph.D., 2008. Lipoic acid prevents high-fat diet induced dyslipidemia and oxidative stress: A microarray analysis. Nutr. 24, 582-588.

Schmidt, T.B., Olson, K.C., Meyer, D.L. \& Brandt, M.M., 2005. Effects of Lipoic Acid supplementation on finishing steer growth performance, carcass merit, beef tenderness and beef retail display properties. Prof. Anim. Scient. 21, 480-485.

StataCorp., 2007. Stata Statistical Software: Release 10. College Station, Texas, U.S.A.

Vignola, G., Lambertini, L., Mazzone, G., Giammarco, M., Tassinari, M., Martelli, G. \& Bertin, G., 2009. Effects of selenium source and level of supplementation on the performance and meat quality of lambs. Meat Sci. 81, 678-685.

Wood, J.D., Enser, M., Fisher, A.V., Nute, G.R., Sheard, P.R., Richardson, R.I., Hughes, S.I. \& Whittington, F.M., 2008. Fat deposition, fatty acid composition and meat quality: A review. Meat Sci. 78, 343-358. 